\title{
Strongly interacting matter under external magnetic fields within nonlocal NJL-type models
}

\author{
V.P. Pagura ${ }^{1, a}$, D. Gómez Dumm ${ }^{2,3}$, S. Noguera ${ }^{1}$, and N.N. Scoccola ${ }^{3,4,5}$ \\ ${ }^{1}$ Departamento de Física Teórica and IFIC, Centro Mixto Universidad de Valencia-CSIC, E-46100 Burjassot \\ (Valencia), Spain \\ ${ }^{2}$ IFLP, CONICET - Departamento de Física, Fac. de Cs. Exactas, Universidad Nacional de La Plata, C.C. \\ 67, 1900 La Plata, Argentina \\ ${ }^{3}$ CONICET, Rivadavia 1917, 1033 Buenos Aires, Argentina \\ ${ }^{4}$ Departamento de Física Teórica, Comisión Nacional de Energía Atómica, Av.Libertador 8250, 1429 Buenos \\ Aires, Argentina \\ ${ }^{5}$ Universidad Favaloro, Solís 453, 1078 Buenos Aires, Argentina
}

\begin{abstract}
We study the behavior of strongly interacting matter under an external magnetic field in the context of nonlocal Nambu-Jona-Lasinio (NJL) -like models. We find that at zero temperature the condensates display the well-known Magnetic Catalysis effect, showing a good quantitative agreement with lattice QCD (LQCD) results. Moreover, when extended to finite temperature we find that (contrary to what happens in the local NJL model) the Inverse Magnetic Catalysis (IMC) effect is naturally incorporated. We also analyze the magnetic susceptibility of the QCD vacuum in the limit of small magnetic field, considering two different model parametrizations, and compare our numerical results to those obtained in other theoretical approaches and in LQCD calculations.
\end{abstract}

\section{Introduction}

Over the last years the understanding of the behavior of strongly interacting matter under magnetic fields has attracted increasing attention due to its relevance for subjects such as the physics of magnetars [1], the analysis of heavy ion collisions at very high energies [2] or the study of the first phases of the Universe [3]. Consequently, considerable work has been devoted to study the structure of the QCD phase diagram in the presence of an external magnetic field [4]. On the basis of the results arising from most low-energy effective models of QCD it was generally expected that, at zero chemical potential, the magnetic field would lead to an enhancement of the chiral condensate ("magnetic catalysis"), independently of the temperature of the system. However, LQCD calculations carried out with physical pion masses $[5,6]$ show that, whereas at low temperatures one finds indeed such an enhancement, the situation is quite different close to the critical chiral restoration temperature: in that region light quark condensates exhibit a nonmonotonic behavior as functions of the external magnetic field, which results in a decrease of the transition temperature when the magnetic field is increased. This effect is known as inverse magnetic catalysis (IMC). Although many scenarios have been considered

ae-mail: Valeria.Pagura@uv.es 
in the last few years to account for the IMC [7-26], the mechanism behind this effect is not yet fully understood. With this motivation, in this work we study the behavior of strongly interacting matter under an external magnetic field in the framework of nonlocal chiral quark models. These theories are proposed as a sort of nonlocal extensions of the well-known NJL model, intending to go a step further towards a more realistic effective approach to QCD. [27-33].

Moreover, these effective models are a useful tool to investigate the QCD vacuum and its nontrivial structure. An interesting aspect to study is the behavior of hadronic systems in the presence of external sources. In particular, it is seen that a constant external electromagnetic field induces the existence of nonvanishing condensates which describe the response of the vacuum to the source. Here, we will study the magnetic susceptibility of the QCD vacuum in the limit of small magnetic field within a nonlocal Polyakov-NJL (PNJL) model that includes wave function renormalization (WFR).

\section{Magnetic catalysis and inverse magnetic catalysis effects}

We begin by stating the Euclidean action for the simplest nonlocal chiral quark model in the case of two light flavours,

$$
S_{E}=\int d^{4} x\left\{\bar{\psi}(x)\left(-i \not \partial+m_{c}\right) \psi(x)-\frac{G}{2} j_{a}(x) j_{a}(x)\right\},
$$

where $m_{c}$ is the current quark mass, assumed to be equal for both flavors. The nonlocal currents are given by $j_{a}(x)=\int d^{4} z \mathcal{G}(z) \bar{\psi}\left(x+\frac{z}{2}\right) \Gamma_{a} \psi\left(x-\frac{z}{2}\right)$ with $\Gamma_{a}=\left(\mathbf{1}, i \gamma_{5} \vec{\tau}\right)$, and $\mathcal{G}(z)$ is a nonlocal form factor that characterizes the effective interaction. In order to study the influence of an external magnetic field we introduce in Eq. (1) a coupling to an electromagnetic (EM) gauge field $\mathcal{A}_{\mu}$. This can be done by introducing appropriate changes in the covariant derivative and also in the nonlocal currents entering the interaction terms. Namely, we perform the changes $\partial_{\mu} \rightarrow \partial_{\mu}-i \hat{Q} \mathcal{A}_{\mu}(x)$, where $\hat{Q}=\operatorname{diag}\left(q_{u}, q_{d}\right)$ and $q_{u}=2 e / 3, q_{d}=-e / 3$ are quark electric charges, together with $\psi(x-z / 2) \rightarrow W(x, x-z / 2) \psi(x-$ $z / 2)$, and related change for $\bar{\psi}(x+z / 2)$. Here $W(s, t)=\mathrm{P} \exp \left[-i \int_{s}^{t} d r_{\mu} \hat{Q} \mathcal{A}_{\mu}(r)\right]$, where $r$ runs over an arbitrary path connecting $s$ with $t$, that, as it is usually done, we take to be a straight line.

We restrict to the case of a constant and homogeneous magnetic field along the 3 -axis, choosing the Landau gauge. Next we perform a standard bosonization of the theory, introducing scalar and pseudoscalar fields $\sigma(x)$ and $\vec{\pi}(x)$. Within the mean field approximation (MFA), pseudoscalar field vacuum expectation values (VEVs) vanish, and we assume the VEV of the scalar field, $\bar{\sigma}$, to be homogeneous in coordinate space. In this way, following the Ritus eigenfunction method [34] we find [35]

$$
\begin{aligned}
& \frac{S_{\mathrm{bos}}^{\mathrm{MFA}}}{V^{(4)}}=\frac{\bar{\sigma}^{2}}{2 G}-N_{c} \sum_{f=u, d} \frac{\left|q_{f} B\right|}{2 \pi} \int \frac{d^{2} \bar{p}}{(2 \pi)^{2}}\left\{\ln \left[\bar{p}^{2}+M_{\bar{p}, 0}^{s_{f}, f^{2}}\right]+\right. \\
& \left.\sum_{k=1}^{\infty} \ln \left[\left(2 k\left|q_{f} B\right|+\bar{p}^{2}+M_{\bar{p}, k}^{-1, f} M_{\bar{p}, k}^{+1, f}\right)^{2}+\bar{p}^{2}\left(M_{\bar{p}, k}^{+1, f}-M_{\bar{p}, k}^{-1, f}\right)^{2}\right]\right\}, \\
& M_{\bar{p}, k}^{\lambda, f}=(-1)^{k-\frac{1-\lambda s_{f}}{2}} \int_{0}^{\infty} d r r \exp \left(-r^{2} / 2\right)\left[m_{c}+\bar{\sigma} g\left(\frac{\left|q_{f} B\right|}{2} r^{2}+\bar{p}^{2}\right)\right] L_{k-\frac{1-\lambda s_{f}}{2}}\left(r^{2}\right) .
\end{aligned}
$$

Here we have defined $\bar{p}=\left(p_{3}, p_{4}\right)$ and $s_{f}=\operatorname{sign}\left(q_{f} B\right)$, while $g\left(p^{2}\right)$ is the Fourier transform of $\mathcal{G}(z), L_{k}(x)$ are the Laguerre polynomials, and the index $k$ labels the Landau levels. We extend this result to finite temperature using the Matsubara formalism. In this way, the corresponding MFA thermodynamical potential $\Omega^{\mathrm{MFA}}$ and the associated gap equation can be obtained. By deriving $\Omega^{\mathrm{MFA}}$ 
with respect to the current quark masses we get the magnetic field dependent quark condensate for each flavor [35],

$$
\begin{aligned}
\left\langle\bar{\psi}_{f} \psi_{f}\right\rangle_{B, T}=-\frac{N_{c}\left|q_{f} B\right| T}{\pi} \int \frac{d p_{3}}{(2 \pi)} \sum_{k=0}^{\infty} \sum_{n=-\infty}^{\infty} \\
\frac{M_{\bar{p}_{n}, k}^{-s_{f}, f}\left[\bar{p}_{n}^{2}+2 k\left|q_{f} B\right|+M_{\bar{p}_{n}, k}^{s_{f}, f^{2}}\right]+(+\leftrightarrow-)}{\left(2 k\left|q_{f} B\right|+\bar{p}_{n}^{2}+M_{\bar{p}_{n}, k}^{-s_{f}, f} M_{\bar{p}_{n}, k}^{s_{f}, f}\right)^{2}+\bar{p}_{n}^{2}\left(M_{\bar{p}_{n}, k}^{s_{f}, f}-M_{\bar{p}_{n}, k}^{-s_{f}, f}\right)^{2}} .
\end{aligned}
$$

This expression is in general divergent. It can be regularized by subtracting and adding a free quark condensate [30], $\left\langle\bar{\psi}_{f} \psi_{f}\right\rangle_{B, T}^{\mathrm{reg}}=\left\langle\bar{\psi}_{f} \psi_{f}\right\rangle_{B, T}-\left\langle\bar{\psi}_{f} \psi_{f}\right\rangle_{B, T}^{\mathrm{free}}+\left\langle\bar{\psi}_{f} \psi_{f}\right\rangle_{B, T}^{\mathrm{free}, \mathrm{reg}}$. In order to make contact with LQCD results quoted in Ref.[6] we introduce the quantity $\Sigma_{B, T}^{f}=\frac{2 m_{c}}{S^{4}}\left[\left\langle\bar{\psi}_{f} \psi_{f}\right\rangle_{B, T}^{\mathrm{reg}}-\left\langle\bar{\psi}_{f} \psi_{f}\right\rangle_{0,0}^{\mathrm{reg}}\right]+1$, where $S=(135 \times 86)^{1 / 2} \mathrm{MeV}$, together with $\Delta \Sigma_{B, T}^{f}=\Sigma_{B, T}^{f}-\Sigma_{0, T}^{f}$ and $\Delta \bar{\Sigma}_{B, T}=\left(\Delta \Sigma_{B, T}^{u}+\Delta \Sigma_{B, T}^{d}\right) / 2$.

To obtain the numerical predictions that follow from the above formalism, it is necessary to specify the particular form of the nonlocal form factor. Here, for simplicity, we consider the case of a Gaussian form factor $g\left(p^{2}\right)=\exp \left(-p^{2} / \Lambda^{2}\right)$. Thus the model has three parameters, $m_{c}, G$, and $\Lambda$, that we fix so as to reproduce empirical values of $f_{\pi}, m_{\pi}$ and a given value of the quark condensate at zero $T$ and $B$, $\Phi_{0} \equiv\left(-\left\langle\bar{\psi}_{f} \psi_{f}\right\rangle_{0,0}^{\text {reg }}\right)^{1 / 3}$. Details can be found in Ref. [31].

Let us analyze numerical results at $T=0$, which are shown in Fig. 1. In the left panel we quote the model predictions for $\Delta \bar{\Sigma}_{B, 0}$ as functions of $e B$ for various model parametrizations, together with results from LQCD [6]. Besides the expected magnetic catalysis effect, we observe that the predictions are very similar for all cases considered and show a very good quantitative agreement with LQCD results. In the right panel we show the corresponding results for $\Sigma_{B, 0}^{u}-\Sigma_{B, 0}^{d}$. We see that, although the overall agreement with LQCD calculations is still good, there is a somewhat larger dependence on the model parametrization.
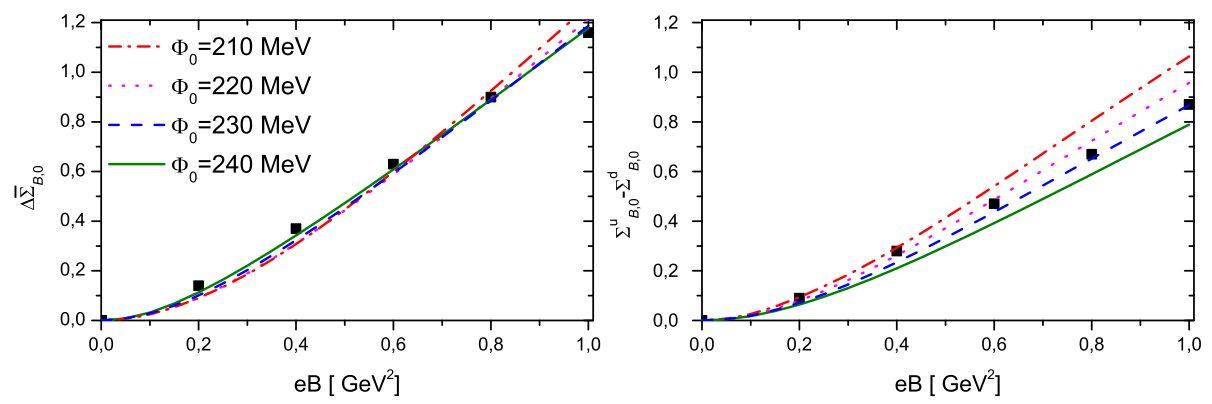

Figure 1. Normalized condensates as functions of the magnetic field at $\mathrm{T}=0$. The curves correspond to different model parametrizations identified by $\Phi_{0}=\left(-\langle\bar{q} q\rangle_{0,0}^{\text {reg }}\right)^{1 / 3}$. Full square symbols correspond to LQCD results of Ref. [6]. Left panel: subtracted flavor average; right panel: flavor difference.

We turn now to our numerical results for the case of finite temperature. In panel (a) of Fig. 2 we quote the values obtained for $\Delta \bar{\Sigma}_{B, T}$ as a function of $e B$, for some representative values of the temperature, while in panel (b) we show the results for $\left(\Sigma_{B, T}^{u}+\Sigma_{B, T}^{d}\right) / 2$ as a function of $T$, for some selected values of $e B$. All values correspond to the parametrization leading to $\Phi_{0}=230 \mathrm{MeV}$, yet 
qualitatively similar results are found for the other parametrizations under consideration. The plots in panel (a) clearly show that, in contrast to what happens at zero temperature, the quantity $\Delta \bar{\Sigma}_{B, T}$ does not display a monotonous increase with $e B$ when one approaches the chiral transition temperature [for this parameter set one has $T_{c}(e B=0)=129.8 \mathrm{MeV}$ ]. In fact, the curves reach a maximum after which $\Delta \bar{\Sigma}_{B, T}$ starts to decrease with increasing $e B$, implying that the present nonlocal model naturally exhibits the IMC effect found in LQCD. This feature can also be seen from the results displayed in panel (b) of Fig. 2. As expected, all curves show a crossover transition from the chiral symmetry broken phase to the (partially) restored one as the temperature increases. However, contrary to what happens e.g. in the standard local NJL model [4], it is seen that within the present model the transition temperature decreases as the magnetic field increases. To be more specific, let us define the critical transition temperature as the value of $T$ at which the derivative $\partial\left[\left(\Sigma_{B, T}^{u}+\Sigma_{B, T}^{d}\right) / 2\right] / \partial T$ reaches a maximum. Since, as known from previous analyses [28, 30, 32], the present model is too simple so as to provide realistic values for the critical temperatures even at vanishing external magnetic field, for comparison with LQCD calculations we consider the relative quantity $T_{c}(B) / T_{c}(0)$. The corresponding results for our four parameterizations are shown in panel (c) of Fig. 2, together with LQCD results from Ref. [6]. From the figure it is clearly seen that for magnetic fields beyond $e B \simeq 0.4 \mathrm{GeV}^{2}$ all parameter sets considered here lead to a decrease of the critical temperature when $e B$ gets increased, i.e. in all cases the IMC effect is observed. On the other hand, the strength of the IMC effect is rather sensitive to the parametrization, the best agreement with LQCD corresponding to the parameter set associated with the lowest value of $\Phi_{0}$ considered here.

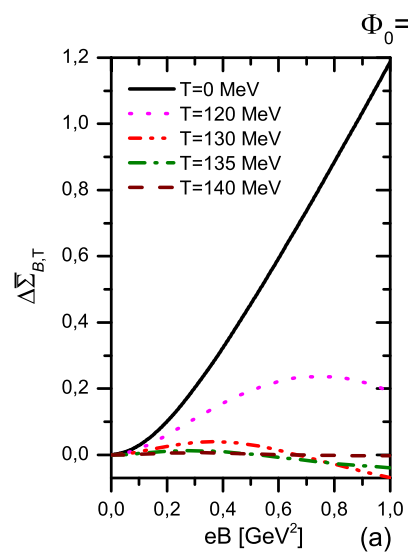

$\Phi_{0}=230 \mathrm{MeV}$
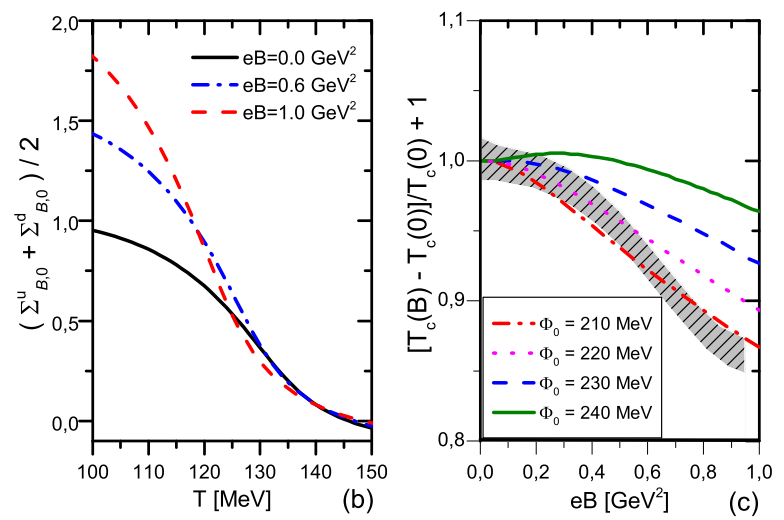

Figure 2. (a) Subtracted normalized flavor average condensate as a function of $e B$ for different representative temperatures. (b) Normalized flavor average condensate as a function of the temperature for different representative values of eB. Results in both panels correspond to $\Phi_{0}=230 \mathrm{MeV}$. (c) Normalized chiral restoration temperatures as functions of $e B$ for various model parametrizations. For comparison, LQCD results of Ref. [6] are indicated by the grey band.

\section{Magnetic susceptibility of the QCD vacuum}

Furthermore, a constant external EM field induces the existence of nonvanishing condensates that characterize the response of the QCD vacuum to the source. We have studied [36], in the context of a 
nonlocal PNJL model that also includes WFR, the VEV of the tensor polarization operator $\left\langle\psi \sigma_{\mu \nu} \psi\right\rangle$, where $\sigma_{\mu \nu}=i\left[\gamma_{\mu}, \gamma_{\nu}\right] / 2$ is the relativistic spin operator. To leading order in the external field one has

$$
\left\langle\bar{\psi}_{f} \sigma_{\mu \nu} \psi_{f}\right\rangle_{A}=q_{f} F_{\mu \nu} \tau_{f},
$$

where $F_{\mu \nu}$ is the field strength tensor and $\tau_{f}$ is the so-called tensor coefficient. In our model, for vanishing temperature, one has [36]

$$
\tau_{f}=4 N_{c} \int \frac{d^{4} p}{(2 \pi)^{4}} Z(p) \frac{M_{f}(p)-p^{2} d M_{f}(p) / d p^{2}}{\left[p^{2}+M_{f}(p)^{2}\right]^{2}} .
$$

Here $M_{f}(p)$ and $Z(p)$ are the (momentum dependent) quark effective mass and WFR, respectively. We have also extended this result to finite $T$, including a coupling of fermions to the Polyakov loop (PL). Different PL potentials found in the literature have been considered [37].

In Fig. 3 we show our results for the normalized $u$ quark tensor coefficient as function of the temperature. In the left panel we present results obtained using an improved polynomial PL potential [37], considering Gaussian (PI) and LQCD-inspired [27] (PII) parametrizations. For comparison we also show the results from Ref. [38], obtained in the context of the instanton liquid model (ILM), as well as LQCD estimates from Ref. [39] (dotted line and grey dashed band, respectively). Defining $T_{c}$ as the temperature corresponding to the inflection point of $\tau_{u}(T)$ we get $T_{c}=158(160) \mathrm{MeV}$ for PI (PII), while LQCD results lead to $T_{c}^{\mathrm{LQCD}} \sim 162 \mathrm{MeV}$ [39]. It is seen that for $T>T_{c}$ the curves obtained within our models are in reasonable agreement with LQCD results. On the other hand the transition shows a steep onset, which could be softened once mesonic fluctuations are included. In the right panel results corresponding to parametrization PII, for various PL potentials. It is found that whereas different potentials give rise to different shapes for $\tau_{u}(T)$ at $T$ below the chiral transition, once the transition is surpassed the functions converge in general to a single curve that is in agreement with LQCD estimates.

\section{Summary}

We have studied the behavior of strongly interacting matter under an external constant magnetic field in the context of nonlocal NJL model. We found that at zero temperature the behavior of the quark condensates shows the expected magnetic catalysis effect, our predictions being in good quantitative agreement with LQCD results. On the other hand, in contrast to what happens in the standard local NJL model, when the analysis is extended to the case of finite temperature our results show that nonlocal models naturally lead to the IMC effect already at the mean field level.

We have also investigated the magnetic susceptibility of the QCD vacuum within a nonlocal PNJL model that includes WFR and we found that the tensor coefficient remains approximately constant up to a critical temperature, at which there is a sudden drop that can be clearly identified with the restoration of the SU(2) chiral symmetry. As occurs for other quantities (e.g. the scalar quark condensates) in the framework of nlPNJL models at the mean field level, we notice that at the onset of the chiral transition the behavior of the tensor coefficient is rather steep in comparison with LQCD estimates. This discrepancy is expected to be cured once meson fluctuations are included in the calculation. In any case, these corrections should not modify the behavior of the tensor coefficient above the transition, which is found to be in good agreement with LQCD results.

Acknowledgements

This work has been supported in part by CONICET and ANPCyT (Argentina), under grants PIP14-492, PIP12-449, and PICT14-03-0492, by the National University of La Plata (Argentina), 

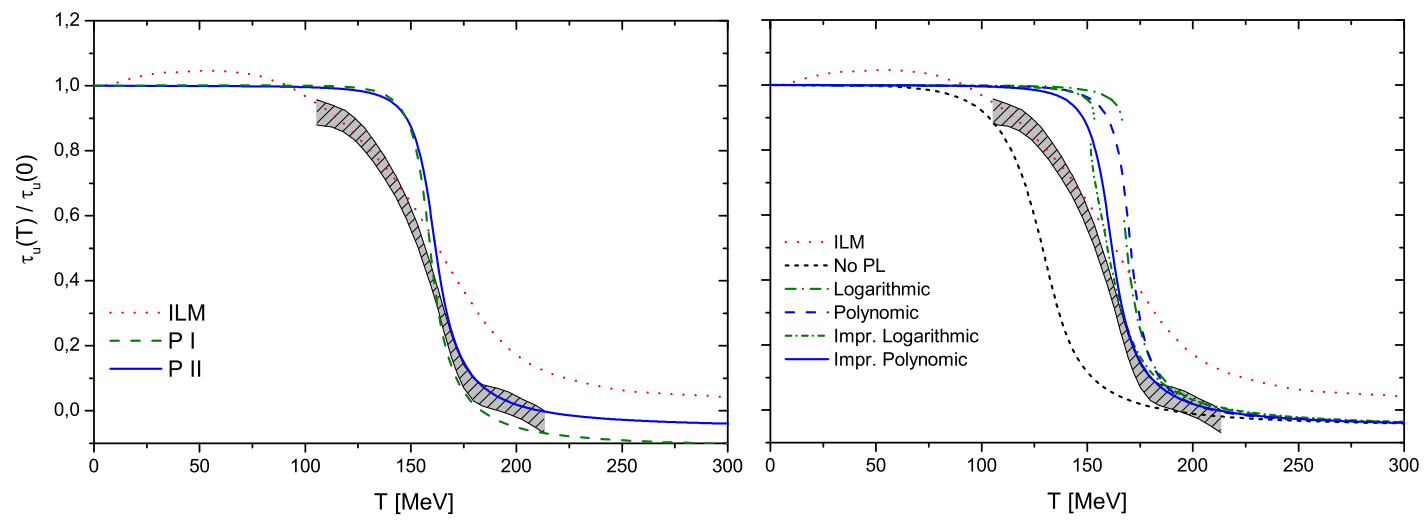

Figure 3. Normalized $u$-quark tensor coefficient vs. temperature for nonlocal PNJL models. Left panel: results corresponding to the improved polynomial PL potential, for parametrizations PI and PII. Right panel: results corresponding to parametrization PII, for various PL potentials. For comparison, values obtained within the ILM [38] (dotted lines) and results from LQCD [39] (dashed grey bands) are also shown in both graphs.

Project No. X718, by the Mineco (Spain) under contract FPA2013-47443-C2-1-P, by the Centro de Excelencia Severo Ochoa Programme grant SEV-2014-0398, and by Generalitat Valenciana (Spain), grant PrometeoII/2014/066.

\section{References}

[1] R. C. Duncan and C. Thompson, Astrophys. J. 392, L9 (1992); C. Kouveliotou et al., Nature 393, 235 (1998).

[2] D. E. Kharzeev, L. D. McLerran and H. J. Warringa, Nucl. Phys. A 803, 227 (2008); V. Skokov, A. Y. Illarionov, and V. Toneev, Int. J. Mod. Phys. A 24, 5925 (2009); V. Voronyuk, V. Toneev, W. Cassing, E. Bratkovskaya, V. Konchakovski, and S. Voloshin, Phys. Rev. C 83, 054911 (2011).

[3] T. Vachaspati, Phys. Lett. B265, 258 (1991); K. Enqvist and P. Olesen, Phys. Lett. B319, 178 (1993).

[4] D. E. Kharzeev, K. Landsteiner, A. Schmitt and H. U. Yee, Lect. Notes Phys. 871, 1 (2013); J. O. Andersen, W. R. Naylor and A. Tranberg, Rev. Mod. Phys. 88, 025001 (2016); V. A. Miransky and I. A. Shovkovy, Phys. Rept. 576, 1 (2015).

[5] G. S. Bali, F. Bruckmann, G. Endrodi, Z. Fodor, S. D. Katz, S. Krieg, A. Schafer and K. K. Szabo, JHEP 1202, 044 (2012).

[6] G. S. Bali, F. Bruckmann, G. Endrodi, Z. Fodor, S. D. Katz and A. Schafer, Phys. Rev. D 86, 071502 (2012).

[7] E. S. Fraga, J. Noronha and L. F. Palhares, Phys. Rev. D 87114014 (2013).

[8] F. Bruckmann, G. Endrodi and T. G. Kovacs, JHEP 1304, 112 (2013).

[9] G. S. Bali, F. Bruckmann, G. Endrodi, F. Gruber and A. Schaefer, JHEP 1304, 130 (2013).

[10] K. Fukushima and Y. Hidaka, Phys. Rev. Lett. 110, 031601 (2013). 
[11] J. Chao, P. Chu and M. Huang, Phys. Rev. D 88, 054009 (2013).

[12] E. S. Fraga, B. W. Mintz and J. Schaffner-Bielich, Phys. Lett. B 731, 154 (2014).

[13] M. Ferreira, P. Costa, D. P. Menezes, C. Providência and N. Scoccola, Phys. Rev. D 89, 016002 (2014); M. Ferreira, P. Costa, O. Lourenço, T. Frederico and C. Providência, Phys. Rev. D 89, 116011 (2014).

[14] A. Ayala, M. Loewe, A. J. Mizher and R. Zamora, Phys. Rev. D 90, 036001 (2014).

[15] R. L. S. Farias, K. P. Gomes, G. I. Krein and M. B. Pinto, Phys. Rev. C 90, 025203 (2014).

[16] A. Ayala, M. Loewe and R. Zamora, Phys. Rev. D 91, 016002 (2015); A. Ayala, C. A. Dominguez, L. A. Hernandez, M. Loewe and R. Zamora, Phys. Rev. D 92, 096011 (2015); Addendum: [Phys. Rev. D 92, 119905 (2015)].

[17] S. Fayazbakhsh and N. Sadooghi, Phys. Rev. D 90, 105030 (2014).

[18] J. O. Andersen, W. R. Naylor and A. Tranberg, JHEP 1502, 042 (2015).

[19] N. Mueller and J. M. Pawlowski, Phys. Rev. D 91, 116010 (2015).

[20] A. Ayala, J. J. Cobos-Martínez, M. Loewe, M. E. Tejeda-Yeomans and R. Zamora, Phys. Rev. D 91, 016007 (2015).

[21] E. J. Ferrer, V. de la Incera and X. J. Wen, Phys. Rev. D 91, 054006 (2015).

[22] J. Braun, W. A. Mian and S. Rechenberger, Phys. Lett. B 755, 265 (2016).

[23] R. Rougemont, R. Critelli and J. Noronha, Phys. Rev. D 93, 045013 (2016).

[24] A. Ayala, C. A. Dominguez, L. A. Hernandez, M. Loewe and R. Zamora, Phys. Lett. B 759, 99 (2016).

[25] S. Mao, Phys. Lett. B 758, 195 (2016).

[26] K. Kashiwa, Phys. Rev. D 83, 117901 (2011).

[27] S. Noguera and N. N. Scoccola, Phys. Rev. D 78, 114002 (2008).

[28] I. General, D. Gomez Dumm and N. N. Scoccola, Phys. Lett. B 506, 267 (2001); D. Gomez Dumm and N.N. Scoccola, Phys. Rev. D 65, 074021 (2002).

[29] A. Scarpettini, D. Gomez Dumm and N.N. Scoccola, Phys. Rev. D 69, 114018 (2004).

[30] D. Gomez Dumm and N. N. Scoccola, Phys. Rev. C 72, 014909 (2005).

[31] D. Gomez Dumm, A. G. Grunfeld and N.N. Scoccola, Phys. Rev. D 74, 054026 (2006).

[32] G. A. Contrera, D. Gomez Dumm and N. N. Scoccola, Phys. Lett. B 661, 113 (2008); G. A. Contrera, M. Orsaria and N. N. Scoccola, Phys. Rev. D 82, 054026 (2010); J. P. Carlomagno, D. Gómez Dumm and N. N. Scoccola, Phys. Rev. D 88, 074034 (2013).

[33] D. Gomez Dumm, S. Noguera and N.N. Scoccola, Phys. Lett. B 698, 236 (2011); Phys. Rev. D 86, 074020 (2012).

[34] V. I. Ritus, Sov. Phys. JETP 48, 788 (1978).

[35] V. P. Pagura, D. Gomez Dumm, S. Noguera and N. N. Scoccola, arXiv:1609.02025 [hep-ph].

[36] V. P. Pagura, D. Gómez Dumm, S. Noguera and N. N. Scoccola, Phys. Rev. D 94, no. 5, 054038 (2016).

[37] C. Ratti, M. A. Thaler and W. Weise, Phys. Rev. D 73, 014019 (2006); S. Roessner, C. Ratti and W. Weise, Phys. Rev. D 75, 034007 (2007); O. Scavenius, A. Dumitru and J. T. Lenaghan, Phys. Rev. C 66, 034903 (2002); L. M. Haas, R. Stiele, J. Braun, J. M. Pawlowski and J. SchaffnerBielich, Phys. Rev. D 87, 076004 (2013).

[38] S. i. Nam, Phys. Rev. D 87, no. 11, 116003 (2013).

[39] G. S. Bali, F. Bruckmann, M. Constantinou, M. Costa, G. Endrodi, S. D. Katz, H. Panagopoulos and A. Schafer, Phys. Rev. D 86, 094512 (2012). 